\title{
Assessment of Maternal Referral Systems in Dr. Sardjito General Hospital Using Phyo Referral Score: A Single- Center Preliminary Study
}

\author{
Eugenius Phyowai Ganap ${ }^{1,2 *}$ Mohammad Hakimi ${ }^{1}$, Ova Emilia ${ }^{2}$ \\ ${ }^{I}$ Division of Social Obstetrics and Gynecology, Department of Obstetrics and Gynecology Dr. Sardjito General \\ ${ }^{2}$ Department of Medical Education, Faculty of Medicine, Public Health and Nursing, Universitas Gadjah Mada,
} Hospital, Indonesia Indonesia

*Corresponding author. Email: phyowai_ku@yahoo.com

\begin{abstract}
Evaluation for referral system of maternal health is a method to decrease maternal mortality rate. Several factors that affecting the quality of referral system are staff, protocols, and geographical access play important roles in the maternal health program. The study aimed to analyze and formulate an evaluation toolkit (Phyo Referral Score/PRS) that can assess the quality of referrals as an instrument for identifying maternal with high-risk complication during labor. This study was qualitative-quantitative (mixed-method) with prospective cohort design which undertaken at Dr. Sardjito General Hospital, Yogyakarta within January and December 2016. The validity, sensitivity, and specificity test were carried out first on the evaluation instrument. Samples were obtained in consecutive patients from obstetric referral at Emergency Department of Dr. Sardjito General Hospital. Statistical analysis was conducted using SPSS v. 19. Statistical analysis was significance if $\mathrm{p}<0.05$. Logical validity test was obtained $93.3 \%(\mathrm{p}=0.001)$ experts agreed to use PRS. For sensitivity and specificity test were $72.7 \%$ and $88.9 \%$ respectively. The results of obstetrics evaluation toolkit trial on 20 subjects, consisting of $11(55 \%)$ of patients were categorized as good quality referrals and $9(45 \%)$ patients as poor quality referrals. The result of bivariate analysis showed that the poor quality referral was associated with 21.33 higher risk for incidence of maternal complication (OR: 21.3; 95\% CI: 1.81-251.26). In conclusion, PRS shows promise as a toolkit to assess the quality of maternal referrals in Dr. Sardjito General Hospital as the highest tertiary referral hospital in Yogyakarta.
\end{abstract}

Keywords: maternal mortality rate, maternal perinatal audit, referral quality, Phyo Referral Score

\section{INTRODUCTION}

Maternal mortality is a major public health concern, particularly in developing country such as Indonesia. In Indonesia, maternal mortality rate have declined by $15 \%$ from 359 deaths per 100.000 live births in 2012 to 305 deaths per 100.000 live births in 2015 [1,2]. The context and causes of maternal mortality and morbidity are well known, and strategies to decrease them were recently implemented. One of the effective strategies is to evaluate and improve maternal health services' referral system. Good quality health referral system is an important element of the WHO Making Pregnancy Safer program [3].

There are several important factors affecting the quality of referral system such as staff, protocols, transportation, communication, and geographical access/distance $[4,5,6,7]$ that need to be concerned by the stakeholders which involved in the maternal and child health program. By evaluating the quality of referrals it can be found which factors that were not working optimally and could be considered for maternal and perinatal audit.

In this study, we aim to analyse and formulate an evaluation toolkit that can assess the quality of referrals as an instrument for identifying maternal with high-risk complication during labor.

\section{METHOD}

This study was a single-center preliminary study which using qualitative-quantitative (mixed-method) with prospective cohort design which undertaken at Dr. Sardjito General Hospital, Yogyakarta within January and December 2016. The validity, sensitivity, and specificity test were carried out first on the evaluation instrument. Samples were obtained in consecutive patients from obstetric referral at Emergency Department of Dr. Sardjito General Hospital. Statistical analysis was conducted using SPSS v. 19 (IBM Corp., NY, USA). Statistical analysis was significance if $\mathrm{p}<0.05$.

The Medical and Health Research Ethics Committee 


\section{Discussion}

This study showed the validity of the Phyo Referral and Dr. Sardjito Hospital had approved the research protocol (Ref: KE/FK/1192/EC/2016).

\section{RESULTS AND DISCUSSION}

\section{Results}

Comprehensive literature review was conducted to find variables or factors which affect maternal health referral system. Several factors were included in this instrument such as age, geographical access, indication for referral, referral staff, initial procedure/treatment prior referral, communication, transportation vehicle, insurance coverage, and distance for referral $[4,5,6,7,8]$

Below was an instrument that we have already formulated entitled Phyo Referral Score (PRS) (Table 1).

Table 1. Phyo Referral Score for assessing quality of maternal health referral system.

\begin{tabular}{|c|c|c|c|c|c|c|}
\hline No & Parameters & & Details & & & Nilai \\
\hline \multirow{4}{*}{1} & \multirow{4}{*}{$\begin{array}{l}\text { Patient and } \\
\text { Cause of } \\
\text { referral }\end{array}$} & Age & $<20$ or $>35$ & $20-35$ & & \\
\hline & & & 0 & 1 & & \\
\hline & & Cause & Avoidable & Unavoidable & & \\
\hline & & & 0 & 1 & & \\
\hline \multirow{10}{*}{2} & \multirow{10}{*}{$\begin{array}{l}\text { Healthcare staff, } \\
\text { Communication } \\
\text { and } \\
\text { Transportation }\end{array}$} & Staff & Non Medic & Medic & & \\
\hline & & & 0 & 1 & & \\
\hline & & Procedure & $\begin{array}{c}\text { Without } \\
\text { stabilization }\end{array}$ & Stabilization & & \\
\hline & & & 0 & 1 & & \\
\hline & & Communication & Without phoned & Phoned & & \\
\hline & & & 0 & 1 & & \\
\hline & & & Non Ambulance & Ambulance & & \\
\hline & & & 0 & 1 & & \\
\hline & & & No Insurance & Insurance & & \\
\hline & & & 0 & 1 & & \\
\hline \multirow{4}{*}{3} & \multirow{4}{*}{$\begin{array}{l}\text { Geographical } \\
\text { Access }\end{array}$} & Area & Different District & Same district & & \\
\hline & & & 0 & 1 & & \\
\hline & & $\begin{array}{l}\text { Distance / } \\
\text { GPS }(\mathrm{km})\end{array}$ & $>30$ & $10-30$ & $10>$ & \\
\hline & & & 0 & 1 & 2 & \\
\hline \multicolumn{6}{|c|}{ TOTAL } & \\
\hline & \multicolumn{2}{|l|}{ Notes for total score: } & & & & \\
\hline & \multicolumn{3}{|c|}{$>5=$ Good quality referral } & & & \\
\hline & \multicolumn{3}{|c|}{$\leq 5=$ Poor quality referral } & & & \\
\hline
\end{tabular}

The criterion validity assessment was conducted using ROC curve of the PRS. The area under the curve was found to be 1,00 which indicates that poor quality maternal referral can be satisfactorily identified using the total score. The suggested cutoff point was 5 points. At cutoff point 5 , we observed that the instrument presented relatively high sensitivity $(72.7 \%)$ and high specificity $(88.9 \%)$.

Table 2. Sensitivity and Specificity test for Phyo Referral Score.

\begin{tabular}{|c|c|c|c|c|c|c|}
\hline $\begin{array}{c}\text { Referra } \\
1 \\
\text { quality }\end{array}$ & $\begin{array}{c}\text { Complication } \\
\mathbf{s} \\
(\mathrm{n}=11)\end{array}$ & $\begin{array}{c}\begin{array}{c}\text { Without } \\
\text { complication } \\
\mathbf{s} \\
(\mathrm{n}=9)\end{array} \\
\end{array}$ & Total & $\begin{array}{c}p- \\
\text { valu } \\
\text { e }\end{array}$ & $\begin{array}{c}\text { OR } \\
\text { (CI } \\
\mathbf{9 5 \% )}\end{array}$ & $\underset{\mathbf{C}}{\mathbf{A U}}$ \\
\hline Poor & $8(88,9 \%)$ & $1(11,1 \%)$ & $\begin{array}{c}9 \\
(100 \% \\
)\end{array}$ & \multirow{2}{*}{$\begin{array}{c}0,01 \\
5\end{array}$} & \multirow{2}{*}{$\begin{array}{c}21,33 \\
\\
(1,81- \\
251,26 \\
)\end{array}$} & \multirow{2}{*}{$\begin{array}{c}1,00 \\
0\end{array}$} \\
\hline Good & $3(27,3 \%)$ & $8(72,7 \%)$ & $\begin{array}{c}11 \\
(100 \% \\
)\end{array}$ & & & \\
\hline
\end{tabular}

Score (PRS) in distinguishing maternal with poor referral quality compared to those with good referral quality. Several parameters which used in the PRS were the most cited "challenges" in maternal health referral system [8].

This study confirmed that this instrument correctly classified $88.9 \%$ of the study subjects with poor referral quality. These data suggest that the PRS is useful and should be included in the protocol for investigating maternal referral quality.

When we tested the criterion validity of the instrument using the 5 cutoff points, we found the sensitivity $(72.7 \%)$ and specificity $(88.9 \%)$, meaning that the PRS is capable of identifying poor referral quality among all maternal referral cases in Dr. Sardjito Hospital during the study period.

There was statistical difference from outcomes between maternal who classified as poor referral quality and good referral quality. The result showed that the poor referral quality was associated 21.33 times higher with the incidence of maternal complication $(\mathrm{OR}=21.33 ; 95 \% \mathrm{CI}=$ 1.81-251.26; $\mathrm{p}=0.015)$. This result was similar with previous study conducted in Jakarta by Purnama et al., (2010) which found that factor which affecting obstetric referral quality may also affect maternal outcomes [9].

Numerous challenges had to be addressed in the context of maternal referral process such as inability to communicate with tertiary referral hospital due to a poor cellular network, distance from the referral hospital, lack of a vehicle or ambulance by the referral institution were commonly cited $[6,10,11]$.

Intervention for improvement of maternal health referral system in the rural settings such as ambulance deployment and also radio call system had proven to be effective to reduce maternal and neonatal health [12].

Until now, Dr. Sardjito Hospital does not have specific records for maternal referral cases. According to our best knowledge, this study is the first study in the hospital that evaluated quality of maternal health referral using scoring system. This can be used as basic data for further studies to improve the quality of maternal health referral.

\section{CONCLUSION}

The data that we have reported in this paper help to establish a baseline data for the development of an effective or good quality of maternal referral system in the Yogyakarta. In conclusion, PRS shows promise as a toolkit to assess the quality of maternal referrals in Dr. Sardjito General Hospital as the highest tertiary referral hospital in Yogyakarta. Since our study was a preliminary study with small study subjects, it needs to be continue with more amount of subjects.

\section{ACKNOWLEDGMENT}

We would like to acknowledge and thank to Aditya Doni Pradana, M.D., for his kind help with the project and protocols document also preparation of the manuscript. 
referral system in developing countries: A narrative review. Informatics in Medicine Unlocked. 2019; 15: 111.

[9] Purnama DI, Madjid OA, Iljanto S. Evaluation of Obstetric Emergency Referral Cases at Dr. Cipto Mangunkusumo Hospital January - December 2008. Indonesia Journal of Obstetrics and Gynecology. 2010; 34(4): 164-169.

[10] Daum L, Konstantynovska O, Solodiankin O, Liashenko O, Poteiko P, Bolotin V, et al. NextGeneration Sequencing for Characterizing Drug Resistant Mycobacterium tuberculosis Genes from Clinical Isolates in the Ukraine. J Clin Microbiol. 2018;(March).

[11] Benson AE, Benson MJ, Luke AH. Assessment of maternal referral systems used for a rural Zambian hospital: the development of setting specific protocols for the identification of complications. African Health Sciences. 2019; 19(1): 1536-1543.

[12] Krasovec K. Auxiliary technologies related to transport and communication for obstetric emergencies. International Journal of Gynaecology and Obstetrics. 2004; 85 (Suppl 1): S14-23.

[13] Hussein J, Kanguru L, Astin M, Munjanja S. The effectiveness of emergency obstetric referral interventions in developing country settings: a systematic review. 2012; 9(7): e1001264. 